\title{
The hydraulic size of a dam: a metric indicating potential hydrologic impact from storage and diversion
}

\begin{abstract}
Easily-calculated metrics describing potential impacts of dams are often used as preliminary investigations, or in analyses describing hydrologic impact over large scales and involving many dams. However, common dam impact metrics overwhelmingly reflect effects of medium to large reservoirs. Indicators applied to describe a dam's capacity to alter downstream river flows typically consider reservoir storage as a sole mechanism of hydrologic impact. Herein, metrics of hydraulic size are proposed to encompass potential impacts related to both storage and diversion of water. Hydrologic effect due to storage and diversion computed across a sample of diversion and non-diversion dams exemplifies that hydrologic alteration related to diversion may be significant, even where storage-based indicators suggest that potential for hydrologic impact is low. As compared to metrics based on storage alone, the proposed metrics offer a more flexible and inclusive description of hydrologic impact and one which is more universally applicable to dams of variable design and purpose.
\end{abstract}

Keywords: Dams, Hydrologic alteration, Diversion, Storage, Impact assessment
Volume I Issue 4 - 2017

\author{
Kibler KM \\ Department of Civil Environmental \& Construction Engineering,
} University of Central Florida, USA

Correspondence: Kibler KM, Department of Civil Environmental \& Construction Engineering, University of Central Florida, USA, Email Kelly.Kibler@ucf.edu

Received: August 25, 2017| Published: November 02, 2017

\section{Introduction}

River scientists and engineers have long sought to understand and quantitatively describe potential for hydro-geomorphic impact to the river environment caused by dams and flow regulation. Despite acknowledgement that simplified metrics (e.g. dam height, reservoir size, installed hydropower capacity, gross static head) are potentially poor indicators of a dam's environmental impact ${ }^{1,2}$ and despite development of more comprehensive models ${ }^{3-5}$ simplified indicators of impact size remain widely referenced. In some cases, for instance in differentiating small and large hydropower dams on the basis of power generation capacity, such indicators are used as policy tools in attempt to segregate high- and low-impact projects. ${ }^{6,7}$ If metrics applied in dam decision making fail to adequately indicate environmental effect size, ${ }^{8}$ outcomes could include outsized and unintended ecological impact, including loss of aquatic biodiversity and eosystem services. However, the persistence of simplified indicators, including those known to be at best reductionist and at worst misleading, speaks to the need for broad-based and simple classification metrics that more accurately indicate the potential hydrologic effect of a dam. Herein, a metric of hydraulic dam size is proposed to fulfill the need for preliminary analysis of potential hydrologic impact. Notably, the proposed non dimensional indicator encompasses multiple potential mechanisms of hydrologic impact, including water storage and diversion, within a single metric. The proposed metric more accurately assesses potential impact from diversion and allows for direct comparison of hydraulic size between dams of varied design and operations, as well as across scales.

Mechanisms of hydrologic impact from dams: The ways in which storage reservoirs affect downstream flow regimes and aquatic ecosystems have been extensively reported and reviewed ${ }^{9-14}$ and metrics indicating potential for hydrologic change by dams $\mathrm{s}^{5-16}$ are often designed to describe such large dam-storage reservoir complexes. For example, the primary mechanism for hydrologic change from large dam-reservoir facilities derives from control supported by reservoir storage ${ }^{17}$ Larger storage capacity relative to inflows may indicate that operations have greater capacity to change the river's natural flow pattern, for instance by storing flood peaks, increasing base flows, or altering the timing and frequency of high and low flows. ${ }^{12-18}$ Hence, metrics such as the ratio of reservoir storage to annual flows ${ }^{10-15}$ or similar derivatives of retention time ${ }^{16}$ are widely applied to describe impact potential. Such metrics elegantly normalize the degree of reservoir storage control by the size of the river on which they are placed. As such, a large reservoir placed on a comparatively large river is gauged to have less flow regulation potential than a reservoir of similar size constructed on a smaller river. Such a normalization of effect to encompass the river's capacity offers substantial improvement over metrics which indicate the size of infrastructure alone (dam height, reservoir volume or area) with no corresponding information regarding river size. However, storage-based metrics will fail to predict hydrologic alteration by diversion, particularly at facilities characterized by comparatively small reservoirs but with large capacity to modify flows through diversion. ${ }^{19}$ Unlike storage reservoirs, hydrologic alteration in the case of diversion dams is not necessarily achieved through reservoir control, but rather by the capacity of diversion. Hydrologic effects from diversion dams and smaller reservoirs have been investigated and reported far less widely, such that the literature developed around hydrologic alteration by dams and dam impact metrics overwhelmingly reflect effects of medium to large reservoirs. However, estimates by Pareto distribution models indicate that globally well over $99 \%$ of existing reservoirs are likely characterized by surface areas less than $1 \mathrm{~km}^{2} .{ }^{12-20}$ Thus, the most commonly applied metrics for indicating downstream hydrologic impact of dams may be systematically biased, identifying impacts related to storage while failing to recognize diversion as another primary mechanism of hydrologic impact. The outcome narrative, 
that a diversion dam with small reservoir has little potential to impact downstream hydrology, is in some case wholly incorrect. ${ }^{1}$ The few existing hydrologic analyses related to diversion hydropower dams, for instance indicate potential for severe hydrologic impact related to diversion. ${ }^{19-24}$ Therefore, metrics that encompasses multiple potential mechanisms of hydrologic alteration may be more universally applicable, and will better facilitate comparisons between dams of diverse purpose and design.

The hydraulic size of a dam: a proposed metric: A non dimensional metric of dam hydraulic size $i(t)_{d a m}$ (Eq. 1) is computed by joint consideration of flow control through both reservoir storage and diversion, normalized relative to river flows:

$$
i(t)_{\text {dam }}=\frac{\max \left\{V(t)_{\text {res }}, \int_{0}^{t} Q(t)_{d i v} d t\right\}}{\int_{0}^{t} Q(t)_{\text {flow }} d t}
$$

In Eq. 1, $V(t)_{r e s}$ is the mean reservoir volume $\left(\mathrm{L}^{3}\right)$ over a given time period, $Q(t)_{\text {flow }}$ and $Q(t)_{\text {div }}$ are respectively the instantaneous river inflows and diverted river flows $\left(\mathrm{L}^{3} \mathrm{t}^{-1}\right)$ over the same time period. The most universally informative time period for analysis is likely annual; however the seasonal variability of dam hydraulic size may also be of interest and hydraulic size may be computed over other durations.

Hydraulic size should be computed using the greater of reservoir volume or diverted volume; to combine the two additively could be misleading, for instance, in the case of irrigation dams, where both storage and diversion are potentially significant sources of alteration. In such cases, additional information about the specific nature of potential hydrologic effect can be discerned in the comparison of the two discrete hydraulic size components, $i(t)_{\text {storage }}$ and $i(t)_{\text {diversion }}$ (Eq. 2a \& 2b).

$$
\begin{aligned}
& i(t)_{\text {storage }}=\frac{V(t)_{\text {res }}}{\int_{0}^{t} Q(t)_{\text {flow }} d t} \\
& i(t)_{\text {diversion }}=\frac{\int_{0}^{t} Q(t)_{\text {div }}}{\int_{0}^{t} Q(t)_{\text {flow }} d t}
\end{aligned}
$$

The two components are characterized by different theoretical upper bounds. The upper bound of $i(t)_{\text {storage }}$ may be over one, as storage of very large reservoirs may exceed annual runoff volume. On the other hand, $i(t)_{\text {diversion }}$ may not exceed one. Comparison of hydraulic size components allows for quantitative description of the relative contributions of storage and diversion to overall alteration potential. For instance, when applied to a set of diversion and non-diversion dams (Figure 1) comparison of discrete hydraulic size components clearly indicates that diversion, rather than storage, is the primary mechanism for hydrologic change below diversion dams. The mean $i(t)_{\text {diversion }}$ across the diversion dams analyzed can be interpreted to convey that $75 \pm 7$ percent of incoming flows may be controlled by diversion, while mean $i(t)_{\text {storage }}$ conveys that less than 0.02 percent of flows may be stored in the reservoirs. Additionally, the capability to compare hydraulic size across the variable sample of dams (this sample includes diversion and non-diversion dams, heights ranging from $4 \mathrm{~m}$ to over $300 \mathrm{~m}$; reservoir volumes ranging from $10^{-3} \mathrm{mcm}$ to over $10^{4} \mathrm{mcm}$ ) provides a conceptual illustration of how different dam designs may affect downstream hydrology. At the analysis scale of individual dams, hydraulic size of only the largest non-diverting storage reservoirs are within the same order of magnitude as all of the diversion dams. Contrarily, if impact potential is computed based on storage only, all diversion dams fall well below the $2 \%$ regulation threshold proposed to indicate potential for significant hydrologic change. ${ }^{12}$ The potential for hydrologic alteration due to diversion clearly can be substantial and should thus be accounted within dam impact indicators.

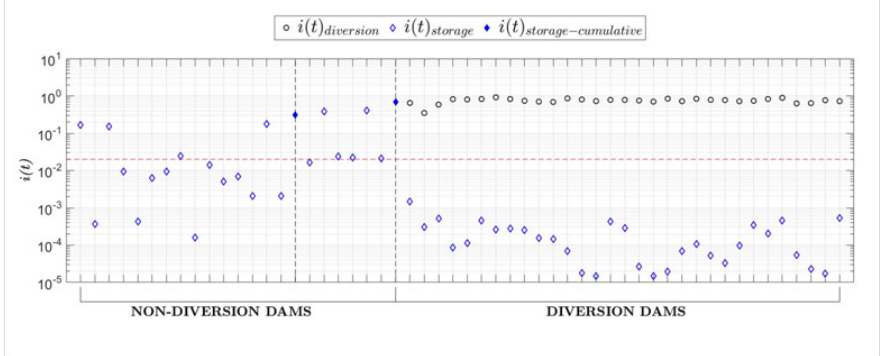

Figure I Hydraulic size components of a sample of diversion and nondiversion dams, with the $2 \%$ regulation threshold indicated data from I, 22 , 23 and 24 .

Hydraulic size analysis nodes: Regulation metrics developed for dams with storage reservoirs are traditionally computed at the dam site using observed or estimated reservoir inflows. Analysis of cumulative regulation effects by multiple dams at the reach or river scale may be computed along a river network, by combining upstream storage volume additively across all upstream reservoirs. ${ }^{15}$ However, it is also possible that a single dam may be linked to more than one location of impact, particularly if water is diverted outside of the river basin. It therefore may be necessary to compute hydraulic size as a measure of flow regime impact from a single dam at multiple analysis nodes; for instance at the site of flow diversion (at the dam) and at other locations representing hydrologic signatures of return flows. As an example, water diverted for hydropower production may be transferred to a power generation facility in a different basin, where water diverted from several rivers may be returned to a single river. In the case where diverted water is released to a different river, the tailrace or location of return flow should also be analyzed as a location for potential hydrologic regime impact. ${ }^{3}$ Water diverted for irrigation also may reemerge as return flows to a different river, and similarly may affect hydrologic signatures. The potential for multiple locations of impact may complicate the analysis of cumulative effect of diversion dams, and it should be noted that diversion volumes may not always be additively combined in the same manner as storage volumes. In the case of rivers developed by series of several diversion dams, thorough understanding of diversion and return flow locations are necessary to create a reach-scale cumulative impact metrics 


\section{Conclusion}

New metrics of hydraulic size are proposed to indicate potential for hydrologic impact of a dam. It is suggested that hydraulic size should encompass both storage and diversion as potential mechanisms of hydrologic effect. Comparison of hydraulic size components due to storage and diversion computed across a sample of diversion and non-diversion dams illustrates that the magnitude of possible diversion effects may be comparable to impacts below large storage reservoirs. Metrics based on storage alone would not be sensitive to detect such effects. The proposed metrics therefore present a more flexible and inclusive description of dam impact, and one which is more universally applicable to dams of variable design and purpose, as compared to metrics based on storage alone. While the proposed metrics may constitute incremental improvement over more simplified metrics, it should be stressed that the primary utility in their application remains that of a preliminary analysis or indicator of potential for hydrologic impact. Detailed site- or reach-scale study of actual flow dynamics with and without regulation is necessary to determine true hydrologic impact. However, the proposed metrics may be useful in determining large-scale impacts of flow regulation through combined storage and diversion. To this point, global-scale analyses of potential for hydrologic change due to dams has been biased towards the sample of dams represented in global databases, which are likely larger, with greater storage potential, relative to the global population of dams. Metrics applied in past global assessments additionally have not accounted for potential hydrologic impacts of diversion. Concerted efforts to better describe the global dam population, for instance through combined database and statistical methods, and application of more comprehensive flow alteration indices may enhance understanding of the impact of flow regulation to global hydrology.

\section{Acknowledgment}

None.

\section{Conflict of interest}

None.

\section{References}

1. Poff NL, Hart DD. How dams vary and why it matters for the emerging science of dam removal. Bio Science. 2002;52(8):1-93.

2. Kibler KM, Tullos DD. Cumulative biophysical impact of small and large hydropower development in Nu River, China. Water Resour Res. 2013;49(6):3104-3118.

3. Grant GE, Schmidt JC, Lewis SL. A geological framework for interpreting downstream effects of dams on rivers. In: Jim EO Connor \& Gordon E Grant (Eds.), A Peculiar River: Geology, Geomorphology, and Hydrology of the Deschutes River, Oregon, AGU, USA; 2003. p. 1-18.

4. McManamay RA, Oigbokie CO, Kao SC, et al. Classification of US hydropower dams by their modes of operation. River Research and Applications. 2016;32(7):1450-1468.

5. Grill G, Lehner B, Lumsdon AE, et al. An index-based framework for assessing patterns and trends in river fragmentation and flow regulation by global dams at multiple scales. Environmental Research Letters. 2015;10(1):1-16.

6. Kibler KM, Tullos DD. Reply to comment on Cumulative biophysical impact of small and large hydropower development in Nu River, China. Water Resources Research. 2014;50(1):760-761.
7. Renewable Energy Policy Network for the 21st Century (REN21). Renewables 2010 global status report. Renewable Energy Policy Network for the 21st Century, France; 2010. p. 1-80.

8. United Nations Framework Convention on Climate Change (UNFCCC/ CCNUCC). Thresholds and criteria for the eligibility of hydroelectric power plants with reservoirs as CDM project activities. CDM Executive Board Report 23, Annex 5, German; $2006 \mathrm{a}$.

9. Pringle CM, Freeman MC, Freeman BJ. Regional Effects of Hydrologic Alterations on Riverine Macrobiota in the New World: TropicalTemperate Comparisons. AIBS Bulletin. 2000;50(9):807-823.

10. Nilsson C, Reidy CA, Dynesius M, et al. Fragmentation and flow regulation of the world's large river systems. Science. 2005;308(5720):405-408.

11. Dynesius M, Nilsson C. Fragmentation and flow regulation of river systems in the northern third of the world. Science. 1994;26(5186):753-762.

12. Lehner B, Reidy Liermann C, Carmen R, et al. High-resolution mapping of the world's reservoirs and dams for sustainable river-flow management. Frontiers in Ecology and the Environment. 2011;9(9):494-502.

13. Graf WL. Dam nation: A geographic census of American dams and their large-scale hydrologic impacts. Water Resources Research. 1999;35(4):1305-1311.

14. Vorosmarty CJ, Sahagian D. Anthropogenic disturbance of the terrestrial water cycle. BioScience. 2000;50(9):753-765.

15. Grill G, Dallaire CO, Chouinard EF, et al. Development of new indicators to evaluate river fragmentation and flow regulation at large scales: A case study for the Mekong River Basin. Ecological Indicators. 2014:45:148-159.

16. Vorosmarty CJ, Sharma KP, Fekete BM, et al. The storage and aging of continental runoff in large reservoir systems of the world. Ambio. 1997;26(4):210-219.

17. Poff NL, Hart DD. How dams vary and why it matters for the emerging science of dam removal. BioScience. 2002.52(8): 659-668.

18. Poff NL, Zimmerman JK. Ecological responses to altered flow regimes: A literature review to inform the science and management of environmental flows. Freshwater Biology. 2010;55(1):194-205.

19. Kibler KM, Alipour M. Flow alteration signatures of diversion hydropower: An analysis of 32 rivers in southwestern China. Ecohydrology. 2017;10(5).

20. Downing JA, Prairie YT, Cole JJ, et al. The global abundance and size distribution of lakes, ponds, and impoundments. Limnology and Oceanography. 2006;51(5):2388-2397.

21. Fantin Cruz I, Pedrollo O, Girard P, et al. Effects of a diversion hydropower facility on the hydrological regime of the Correntes River, a tributary to the Pantanal floodplain, Brazil. Journal of Hydrology. 2015;531:10-820.

22. Plinston D, He DM. Water resources and hydropower: Policies and strategies for sustainable development of the Lancang-Mekong River Basin, Rep. TA-3139. Asian Development Bank, Philippines. 1999.

23. Dore J, Yu XG. Yunnan hydropower expansion: Update on China's energy industry reforms and the $\mathrm{Nu}$, Lancang and Jinsha hydropower dams. Chiang Mai University and Green Watershed, Thailand; 2004. p. $1-38$.

24. Kummu M, Varis O. Sediment-related impacts due to upstream reservoir trapping, the Lower Mekong River. Geomorphology. 2007;85(3-4):275-293. 